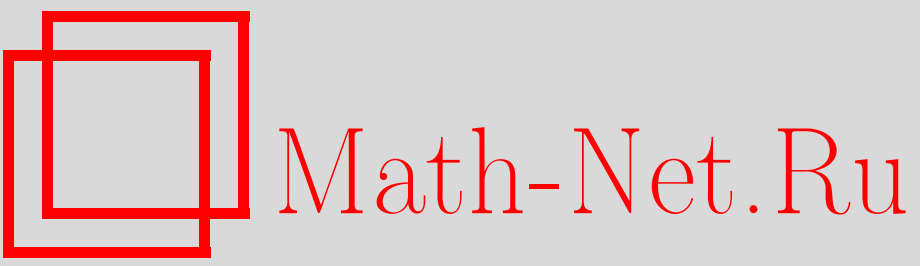

В. Г. Звягин, М. В. Казначеев, Аттракторы для автономной модели движения нелинейно-вязкой жидкости, Итоги науки и техн. Сер. Соврем. мат. и ее прил. Темат. обз., 2021, том 191, 74-91

DOI: https://doi.org/10.36535/0233-6723-2021-191-74-91

Использование Общероссийского математического портала Math-Net.Ru подразумевает, что вы прочитали и согласны с пользовательским соглашением

http: //www.mathnet.ru/rus/agreement

Параметры загрузки:

IP : 54.209 .52 .79

26 апреля 2023 г., 08:39:47 
ИТОГИ НАУКИ И ТЕХНИКИ.

Современная математика и ее приложения.

Тематические обзоры.

Том 191 (2021). С. 74-91

DOI: 10.36535/0233-6723-2021-191-74-91

УДК 517.958

\title{
АТТРАКТОРЫ ДЛЯ АВТОНОМНОЙ МОДЕЛИ ДВИЖЕНИЯ НЕЛИНЕЙНО-ВЯЗКОЙ ЖИДКОСТИ
}

\author{
(c) 2021 г. $\quad$ В. Г. ЗВЯГИН, М. В. КАЗНАЧЕЕВ
}

\begin{abstract}
АннотАция. В работе изучается предельное поведение слабых решений автономной модели движения нелинейно-вязкой жидкости, в ситуации, когда время стремится к бесконечности. А именно, для решений рассматриваемой модели установлено существование слабых решений на положительной полуоси, определено траекторное пространство, соответствующее решениям этой модели, и на основе теории траекторных пространств доказано существование вначале минимального траекторного аттрактора, а затем и глобального аттрактора в фазовом пространстве. Таким образом, оказывается, что каково бы ни было начальное состояние системы, описывающей рассматриваемую модель, с течением времени оно «забывается» и неограниченно приближается к глобальному аттрактору.
\end{abstract}

Ключевые слова: аттрактор, траекторное пространство, нелинейно вязкая жидкость, слабое решение.

\section{ATTRACTORS FOR AN AUTONOMOUS MODEL OF THE MOTION OF A NONLINEAR VISCOUS FLUID}

\author{
(c) 2021 V. G. ZVYAGIN, M. V. KAZNACHEEV
}

\begin{abstract}
In this paper, we examine the limit behavior of weak solutions of an autonomous model of motions of a nonlinear viscous fluid, in the case where the time tends to infinity. Namely, for solutions of the model considered, the existence of weak solutions on the positive semiaxis is proved, the corresponding trajectory space the model is introduced, and the existence of the minimal trajectory attractor and the global attractor in the phase space is proved. Thus, it turns out that any initial state of the system approaches to the global attractor.
\end{abstract}

Keywords and phrases: attractor, trajectory space, nonlinear viscous fluid, weak solution.

AMS Subject Classification: 35B41

1. Введение. Эволюционные уравнения математической физики и, в частности, гидродинамики можно истолковывать как законы эволюции некоторых систем. Математическое описание системы включает в себя некоторое множество, называемое фазовым пространством, элементы которого отождествляются с состояниями системы, и закон эволюции, который показывает, как состояние меняется со временем.

При изучении динамики систем особый интерес представляет их предельное поведение, когда время стремится к бесконечности. На практике часто встречаются системы со следующим свойством: «на бесконечности» их динамика сосредотачивается на небольшой части фазового

Работа выполнена при поддержке Российского научного фонда (проект № 19-11-00146). 
пространства, называемой глобальным аттрактором. Каким бы ни было начальное состояние системы, со временем оно «забывается», и состояние системы неограниченно приближается к глобальному аттрактору. В случае таких систем естественно изучать динамику именно вблизи глобальных аттракторов, поскольку иные состояния заведомо «преходящи». Это причина того, что изучение существования и свойств глобальных аттракторов актуально для математических проблем современного естествознания и, в частности, для гидродинамики.

Понятие «аттрактор» возникло в теории динамических систем, которая изначально была тесно связана с теорией обыкновенных дифференциальных уравнений. В этой теории динамика систем обычно описывается с помощью полугруппы эволюционных операторов. Пусть $E-$ некоторое множество. Семейство отображений $\left\{S_{t}: E \rightarrow E\right\}_{t \geqslant 0}$ называется полугруппой, если выполнены следующие условия:

$$
S_{t} S_{\tau}=S_{t+\tau} \quad(t, \tau \geqslant 0) ; \quad S_{0}=\mathrm{id} .
$$

Поясним, каким образом динамические системы возникают при изучении эволюционных уравнений. Пусть имеется некоторое автономное уравнение

$$
v^{\prime}=A(v) \quad(v \in E)
$$

(автономность означает, что оператор $A$ не зависит от времени). Предположим, что для каждого $v_{0} \in E$ существует единственное решение $v: \mathbb{R}_{+} \rightarrow E$ уравнения (1.1) с начальным условием $v(0)=v_{0}$ (здесь и далее $\left.\mathbb{R}_{+}=[0,+\infty)\right)$. Тогда можно определить операторы сдвига по траекториям уравнения $(1.1)$, положив $S_{t} v_{0}=v(t)(t \geqslant 0)$. С учётом автономности уравнения полугрупповое свойство для семейства $\left\{S_{t}\right\}$ операторов проверяется тривиально.

Отметим, что в случае уравнений математической физики фазовое пространство обычно бесконечномерно.

Классическим приложением теории динамических систем в задачах гидродинамики является доказательство О. А. Ладыженской существования глобального аттрактора двумерной системы Навье-Стокса в [9] (см. также обзорную работу [10]).

Как было отмечено выше, для применения теории аттракторов динамических систем требуется однозначная глобальная разрешимость задачи с заданным начальным условием. Это приводит к существенным трудностям при исследовании многих задач гидродинамики. Для большого числа моделей гидродинамики не доказана глобальная разрешимость в сильном смысле, и, таким образом, сильные решения не могут быть использованы для изучения долговременной динамики. При этом может быть установлена глобальная разрешимость в слабом смысле. Но, как правило, единственность слабого решения с заданным начальным условием также не удаётся установить. Соответственно, нельзя корректно определить полугруппу операторов сдвига по траекториям.

Все эти трудности в полной мере обнаруживаются уже в случае трёхмерной системы НавьеСтокса: для неё не установлено ни существования глобального сильного решения, ни единственности слабого.

Для доказательства существования аттракторов уравнений в частных производных в работах М. И. Вишика и В. В. Чепыжова $[1,2]$ был предложен подход, основанный на рассмотрении траекторных пространств и траекторных аттракторов. Независимо близкий метод был предложен Дж. Селлом в $[15,16]$. Основная идея их теорий состоит в том, чтобы рассматривать слабые решения на $\mathbb{R}_{+}$как точки некоторого множества $\mathcal{H}^{+}$, содержащегося в пространстве функций времени со значениями в банаховом пространстве. На этом множестве рассматривается полугруппа трансляций $\{T(t)\}_{t \geqslant 0}$, действующих по правилу

$$
T(t) g(s)=g(s+t)
$$

Ясно, что полугруппа трансляций может действовать на пространстве $\mathcal{H}^{+}$, если оно трансляционно инвариантно, т.е. $T(t) \mathcal{H}^{+} \subset \mathcal{H}^{+}$при $t \geqslant 0$. В таком случае к полугруппе $\{T(t)\}$ можно применять аналоги методов теории динамических систем.

В теории Вишика-Чепыжова-Селла предложен новый способ описания динамики на множестве: не с помощью задания полугруппы, а с помощью задания пространства траекторий. Отдельные траектории при этом представляют конкретные сценарии развития системы. С точки зрения описания динамики пространство траекторий является более гибким инструментом, 
чем полугруппа операторов. В частности, пространство траекторий позволяет обойти требование детерминированности: не запрещается наличие двух сценариев развития с одним начальным условием. В принципе, пространства траекторий могут не быть связанными с какими-либо уравнениями.

Таким образом, с помощью этого подхода удалось изучить аттракторы трехмерной системы Навье-Стокса (см. [2]), однако в различных задачах гидродинамики условие трансляционной инвариантности пространства траекторий оказывается ограничительным. Дело в том, что траекторные пространства, соответствующие рассматриваемым моделям, строятся на основе некоторых аналогов энергетических оценок, получение которых может представлять значительные технические сложности, и далеко не всегда удаётся получить трансляционно инвариантные оценки. В связи с этим появилась необходимость развития теории аттракторов траекторных пространств для случая трансляционной неинвариантности пространства траекторий, что и удалось сделать в [20] для автономного случая, в [21] для случая равномерных аттракторов и в [19] для пулбекаттракторов (другими словами, обратных аттракторов) траекторных пространств, не удовлетворяющих условию трансляционной инвариантности. Эти аттракторы несколько отличаются от аттракторов трансляционно инвариантных траекторных пространств, в частности, они могут состоять не только из траекторий траекторных пространств и их сечений, но из некоторых других элементов, подробнее об этом можно узнать в $[4,8,23]$. Используя теорию аттракторов трансляционно неинвариантных траекторных пространств, удалось установить существование аттракторов у большого числа моделей неньютоновой гидродинамики (см. [7, 20-22]). В настоящей работе рассматривается автономная модель движения нелинейно-вязкой жидкости, и для неё на основе трансляционно неинвариантных траекторных пространств устанавливается существование траекторного и глобального аттракторов.

2. Описание используемых понятий модели движения и теории аттракторов. Формулировка основного результата. Приведем основные понятия и результаты теории аттракторов неинвариантных траекторных пространств (см. $[8,23])$. Рассмотрим два банаховых пространства $E, E_{0}$, причем $E$ рефлексивно и непрерывно вложено в $E_{0}$.

Пространство $C\left(\mathbb{R}_{+} ; E_{0}\right)$ состоит из непрерывных функций, определенных на $\mathbb{R}_{+}$и принимающих значения в пространстве $E_{0}$. Данное пространство - метрическое. Метрика задается формулой $\rho(u, v)=\|u-v\|_{C\left(\mathbb{R}_{+} ; E_{0}\right)}$, где

$$
\|u\|_{C\left(\mathbb{R}_{+} ; E_{0}\right)}=\sum_{k=1}^{\infty} 2^{-k} \frac{\|u\|_{C\left(0, k ; E_{0}\right)}}{1+\|u\|_{C\left(0, k ; E_{0}\right)}} .
$$

Пространство $L_{\infty}\left(\mathbb{R}_{+} ; E\right)$ состоит из функций $u$, определенных почти всюду на $\mathbb{R}_{+}$и принимающих значения в $E$, для которых найдется число $\alpha(u)$ такое, что $\|u\|_{E} \leqslant \alpha(u)$ при почти всех $t \in \mathbb{R}_{+}$. Норма в $L_{\infty}\left(\mathbb{R}_{+} ; E\right)$ определяется равенством

$$
\|u\|_{L_{\infty}\left(\mathbb{R}_{+} ; E\right)}=\underset{t \in \mathbb{R}_{+}}{\operatorname{vrai} \max }\|u(t)\|_{E} .
$$

Рассмотрим непустое семейство функций $\mathcal{H}^{+} \subset C\left(\mathbb{R}_{+} ; E_{0}\right) \cap L_{\infty}\left(\mathbb{R}_{+} ; E\right)$. Множество $\mathcal{H}^{+}$будем называть пространством траекторий, а его элементы - траекториями.

Дадим основные определения.

Определение 2.1. Множество $\mathcal{A} \subset E$ называется глобальным аттрактором (в $\left.E_{0}\right)$ пространства траекторий $\mathcal{H}^{+}$, если оно удовлетворяет следующим условиям:

(1) множество $\mathcal{A}$ компактно в $E_{0}$ и ограниченно в $E$;

(2) для всякого ограниченного в $L_{\infty}\left(\mathbb{R}_{+} ; E\right)$ множества $B \subset \mathcal{H}^{+}$выполняется условие притягивания

$$
\sup _{u \in B} \inf _{y \in \mathcal{A}}\|u(t)-y\|_{E_{0}} \rightarrow 0 \quad(t \rightarrow \infty)
$$

(3) множество $\mathcal{A}$ является наименьшим по включению, удовлетворяющим условиям (1) и (2).

Определение 2.2. Множество $\mathcal{U} \subset C\left(\mathbb{R}_{+} ; E_{0}\right) \cap L_{\infty}\left(\mathbb{R}_{+} ; E\right)$ называется траекторным аттрактором (пространства траекторий $\mathcal{H}^{+}$), если оно удовлетворяет следующим условиям: 
(1) множество $\mathcal{U}$ компактно в $C\left(\mathbb{R}_{+} ; E_{0}\right)$ и ограниченно в $L_{\infty}\left(\mathbb{R}_{+} ; E\right)$;

(2) имеет место равенство $T(t) \mathcal{U}=\mathcal{U}$ для всех $t \geqslant 0$;

(3) множество $\mathcal{U}$ является притягивающим, т.е. для любого множества $B \subset \mathcal{H}^{+}$, ограниченного в $L_{\infty}\left(\mathbb{R}_{+} ; E\right)$, выполняется условие

$$
\sup _{u \in B} \inf _{v \in \mathcal{U}}\|T(h) u-v\|_{C\left(\mathbb{R}_{+} ; E_{0}\right)} \rightarrow 0 \quad(h \rightarrow \infty) .
$$

Определение 2.3. Минимальным траекторным аттрактором пространства траекторий $\mathcal{H}^{+}$ называется наименьший по включению траекторный аттрактор, т.е. такой аттрактор, который содержится в любом другом траекторном аттракторе.

Определение 2.4. Множество $P \subset C\left(\mathbb{R}_{+} ; E_{0}\right) \cap L_{\infty}\left(\mathbb{R}_{+} ; E\right)$ называется поглощающим для $\mathcal{H}^{+}$, если для каждого множества $B \subset \mathcal{H}^{+}$, ограниченного в норме $L_{\infty}\left(\mathbb{R}_{+} ; E\right)$, найдется такое $t_{B} \geqslant 0$, что $T(t) B \subset P$ при $t \geqslant t_{B}$.

Теорема 2.1. Пусть существует компактное в $C\left(\mathbb{R}_{+} ; E_{0}\right)$ и ограниченное в $L_{\infty}\left(\mathbb{R}_{+} ; E\right)$ поглощающее множество $P$ для пространства траекторий $\mathcal{H}^{+}$. Тогда существует минимальный траекторный аттрактор $\mathcal{U}$ пространства траекторий $\mathcal{H}^{+}$.

Наконец, сформулируем основную теорему о существовании глобального аттрактора неинвариантного пространства траекторий.

Теорема 2.2. Пусть существует минимальный траекторный аттрактор $\mathcal{U}$ пространства траекторий $\mathcal{H}^{+}$. Тогда существует глобальный аттрактор (в $\left.E_{0}\right) \mathcal{A}$ пространства траекторий $\mathcal{H}^{+}$, и справедливо равенство

$$
\mathcal{A}=\mathcal{U}(t), \quad t \geqslant 0 .
$$

Рассмотрим начально-краевую задачу рассматриваемой модели движения нелинейно-вязкой среды. Пусть $\Omega$ ограниченная область в $\mathbb{R}^{n}, n=2,3$, с липшицевой границей $\partial \Omega, T>0$ - заданное число. Движение нелинейно-вязкой несжимаемой жидкости в $Q_{T}:=\Omega \times[0, T]$ описывается уравнениями:

$$
\begin{gathered}
\frac{\partial v(t, x)}{\partial t}+\sum_{i=1}^{n} v_{i}(t, x) \frac{\partial v(t, x)}{\partial x_{i}}-\operatorname{Div}\left\{2 \phi\left(I_{2}(v(t, x))\right) \varepsilon(v(t, x))\right\}+\operatorname{grad} p(t, x)=f(t, x), \\
\operatorname{div} v=0, \quad(t, x) \in Q_{T} ; \quad v(0, x)=v^{0}, \quad x \in \Omega ; \quad v(t, x)=0 \quad x \in[0, T] \times \partial \Omega .
\end{gathered}
$$

Здесь $v=\left(v_{1}(t, x), \ldots, v_{n}(t, x)\right), n=2,3$, и $p(t, x)$ - вектор-функция скорости и давления среды соответственно, $f(t, x)$ - плотность внешних сил. Заданная непрерывно дифференцируемая скалярная функция $\phi$, определенная на интервале $[0, \infty)$, характеризует вязкость. Следуя [11], введем следующие ограничения на функцию $\phi$ :

$$
\begin{gathered}
0<m_{1} \leqslant \phi(s) \leqslant M_{1}<\infty, \quad s \in[0, \infty) ; \\
\left|s \phi^{\prime}(s)\right| \leqslant M_{2}<\infty, \quad s \in[0, \infty) ; \\
-s \phi^{\prime}(s) \leqslant \phi(s) \text { при } \phi^{\prime}(s)<0 .
\end{gathered}
$$

Далее, в системе $(2.1)$ величина $\varepsilon(v)=\left\{\varepsilon_{i j}(v)\right\}_{i, j=\overline{1, n}}$ - тензор скоростей деформаций,

$$
\varepsilon_{i j}(v)=\frac{1}{2}\left(\frac{\partial v_{i}}{\partial x_{j}}+\frac{\partial v_{j}}{\partial x_{i}}\right)
$$

а $I_{2}(v)$ определяется равенством

$$
I_{2}^{2}(v)=\varepsilon(v): \varepsilon(v)=\sum_{i, j=1}^{n} \varepsilon_{i j}^{2}(v) .
$$

Здесь для произвольных квадратных матриц $A=\left(a_{i j}\right)$ и $B=\left(b_{i j}\right)$ используется символ

$$
A: B=\sum_{i, j=1}^{n} a_{i j} b_{i j}
$$


и символ $\operatorname{Div} C$, обозначающий дивергенцию тензора $C=\left(c_{i j}(t, x)\right)$, т.е. вектор

$$
\operatorname{Div} C=\left(\sum_{j=1}^{n} \frac{\partial c_{1 j}(t, x)}{\partial x_{j}}, \ldots, \sum_{j=1}^{n} \frac{\partial c_{n j}(t, x)}{\partial x_{j}}\right) .
$$

Через $C_{0}^{\infty}(\Omega)^{n}$ будем обозначать пространство вектор-функций на $\Omega$ со значениями в $\mathbb{R}^{n}$ класса $C^{\infty}$ с компактным носителем, содержащимся в $\Omega$. Пусть

$$
\mathcal{V}=\left\{v: v \in C_{0}^{\infty}(\Omega)^{n}, \operatorname{div} v=0\right\}
$$

- подмножество соленоидальных вектор-функций; $H$-замыкание $\mathcal{V}$ по норме пространства $L_{2}(\Omega)^{n} ; V$ - замыкание $\mathcal{V}$ по норме пространства $W_{2}^{1}(\Omega)^{n}$. Пространство $V$ вложено в $H$ и плотно в нем, причем вложение непрерывно. Отождествляя, далее, по теореме Рисса $H$ и $H^{*}$, мы приходим к включениям $V \subset H \equiv H^{*} \subset V^{*}$, где каждое пространство плотно в последующем и вложения непрерывны (см. [12, гл. III]).

Введем пространство, в котором будет доказана теорема существования слабых решений

$$
W_{2,1}=\left\{v: v \in L_{2}(0, T ; V) \cap L_{\infty}(0, T ; H), v^{\prime} \in L_{1}\left(0, T ; V^{*}\right)\right\} .
$$

Пространство $W_{2,1}$ снабжено нормой

$$
\|v\|_{W_{2,1}}=\|v\|_{L_{2}(0, T ; V)}+\|v\|_{L_{\infty}(0, T ; H)}+\left\|v^{\prime}\right\|_{L_{1}\left(0, T ; V^{*}\right)} .
$$

Через $\langle f, \varphi\rangle$ будем обозначать действие функционала $f \in X^{*}$ на элемент $\varphi \in X$. Также отметим, что согласно [12, гл. III, лемма 1.1] имеет место равенство

$$
\left\langle v^{\prime}, \varphi\right\rangle=\frac{d}{d t}(v, \varphi), \quad \varphi \in V .
$$

Определение 2.5. Пусть $v^{0} \in H, f \in L_{2}\left(0, T ; V^{*}\right)$. Слабым решением задачи (2.1)-(2.2) на отрезке $[0, T]$ называется функция $v \in W_{2,1}$, удовлетворяющая соотношению

$$
\left\langle v^{\prime}, \varphi\right\rangle-\int_{\Omega} \sum_{i, j=1}^{n} v_{i} v_{j} \frac{\partial \varphi_{j}}{\partial x_{i}} d x+2 \int_{\Omega} \psi\left(I_{2}(v)\right) \varepsilon(v): \varepsilon(\varphi) d x=\langle f, \varphi\rangle,
$$

при всех $\varphi \in V$, п. в. $t \in[0, T]$, и начальному условию

$$
\left.v\right|_{t=0}=v^{0} .
$$

Замечание 2.1. Известно (см. [12, гл. III, леммы 1.11 .4$]$ ), что $W_{2,1} \subset C_{\omega}(0, T ; H)$ - пространство слабо непрерывных функций на $[0, T]$ со значениями в $H$. Следовательно, начальное условие на функцию $v$ имеет смысл.

Сформулируем основной результат данной статьи.

Теорема 2.3. Пусть $f \in V^{*}$. Тогда существует глобальный аттрактор $\mathcal{A}$ пространства траекторий $\mathcal{H}^{+}$и выполняется соотношение

$$
\mathcal{A}=\mathcal{U}(t), \quad t \geqslant 0
$$

3. Операторная трактовка задачи. Дадим операторную трактовку рассматриваемой задачи. Введем операторы при помощи следующих равенств:

$$
\begin{array}{llll}
K: V \rightarrow V^{*}, \quad\langle K(v), \varphi\rangle & =\int_{\Omega} \sum_{i, j=1}^{n} v_{i} v_{j} \frac{\partial \varphi_{j}}{\partial x_{i}} d x, & v \in V, & \varphi \in V ; \\
D: V \rightarrow V^{*}, & \langle D(v), \varphi\rangle=2 \int_{\Omega} \phi\left(I_{2}(v)\right) \varepsilon(v): \varepsilon(\varphi) d x, & v \in V, \quad \varphi \in V .
\end{array}
$$

Тогда из (2.4) получаем операторное уравнение, разрешимость которого, эквивалентна разрешимости задачи (2.4): (2.5)

$$
v^{\prime}(t)+D(v)-K(v)=f(t) .
$$


Таким образом, слабое решение задачи $(2.1)$, (2.2) - это решение $v \in W_{2,1}$ операторного уравнения (3.1), удовлетворяющее начальному условию

$$
\left.v\right|_{t=0}=v^{0} .
$$

Отметим некоторые свойства введенных выше операторов.

Лемма 3.1. Пусть $n \leqslant 4$. Тогда отображсние $K: L_{2}(0, T ; V) \rightarrow L_{1}\left(0, T ; V^{*}\right)$ непрерывно, и справедлива оченка

$$
\|K(v(t))\|_{L_{1}\left(0, T ; V^{*}\right)} \leqslant C_{0}\|u\|_{L_{2}(0, T ; V)}^{2} .
$$

Доказательство данного факта приведено в [12, гл. II, лемма 1.1].

Лемма 3.2. В случае произвольного п отображение $D: L_{2}(0, T ; V) \rightarrow L_{2}\left(0, T ; V^{*}\right)$ непреръвно и монотонно.

Доказательство. Докажем непрерывность оператора $D$. Положив $z=v-u$ и, используя теорему Лагранжа на интервале $[0,1]$ для функции $f(\delta)=\phi\left(I_{2}(u+\delta z)\right) \varepsilon(u+\delta z): \varepsilon(w)$, получим:

$$
\begin{gathered}
\langle D(v)-D(u), w\rangle= \\
=2 \int_{\Omega}\left[\phi\left(I_{2}(v)\right) \varepsilon(v)-\phi\left(I_{2}(u)\right) \varepsilon(u)\right]: \varepsilon(w) d x=2 \int_{\Omega} \frac{d}{d \delta}\left[\phi\left(I_{2}\left(u+\delta_{0} z\right)\right) \varepsilon\left(u+\delta_{0} z\right)\right]: \varepsilon(w) d x= \\
=2 \int_{\Omega}\left[\phi\left(I_{2}\left(u+\delta_{0} z\right)\right) \varepsilon(z)+\frac{d}{d \delta} \phi\left(I_{2}\left(u+\delta_{0} z\right)\right) \varepsilon\left(u+\delta_{0} z\right)\right]: \varepsilon(w) d x= \\
=2 \int_{\Omega}\left[\phi\left(I_{2}\left(u+\delta_{0} z\right)\right) \varepsilon(z): \varepsilon(w)+\frac{\varepsilon\left(u+\delta_{0} z\right): \varepsilon(z)}{\left(\varepsilon\left(u+\delta_{0} z\right): \varepsilon\left(u+\delta_{0} z\right)\right)^{1 / 2}} \frac{d \phi\left(I_{2}\left(u+\delta_{0} z\right)\right)}{d I_{2}\left(u+\delta_{0} z\right)} \varepsilon\left(u+\delta_{0} z\right): \varepsilon(w)\right] d x= \\
=2 \int_{\Omega}\left[\phi\left(I_{2}\left(u+\delta_{0} z\right)\right) \varepsilon(z): \varepsilon(w)+\right. \\
\left.\quad+\frac{1}{I_{2}\left(u+\delta_{0} z\right)} \frac{d \phi\left(I_{2}\left(u+\delta_{0} z\right)\right)}{d I_{2}\left(u+\delta_{0} z\right)}\left(\varepsilon\left(u+\delta_{0} z\right): \varepsilon(z)\right)\left(\varepsilon\left(u+\delta_{0} z\right): \varepsilon(w)\right)\right] d x .
\end{gathered}
$$

Тогда

$$
\begin{aligned}
& |\langle D(v)-D(u), w\rangle| \leqslant \\
& \leqslant 2\left|\int_{\Omega} \phi\left(I_{2}\left(u+\delta_{0} z\right)\right) \varepsilon(z): \varepsilon(w) d x\right|+2\left|\int_{\Omega} I_{2}\left(u+\delta_{0} z\right) \frac{d \phi\left(I_{2}\left(u+\delta_{0} z\right)\right)}{d I_{2}\left(u+\delta_{0} z\right)} I_{2}(z) I_{2}(w) d x\right| \leqslant \\
& \leqslant 2 M_{1}\left(\int_{\Omega} I_{2}^{2}(z) d x\right)^{1 / 2}\left(\int_{\Omega} I_{2}^{2}(w) d x\right)^{1 / 2}+2 M_{2}\left(\int_{\Omega} I_{2}^{2}(z) d x\right)^{1 / 2}\left(\int_{\Omega} I_{2}^{2}(w) d x\right)^{1 / 2} \leqslant \\
& \leqslant\left(2 M_{1}+2 M_{2}\right)\|z\|_{L_{2}(\Omega)^{n}}\|w\|_{L_{2}(\Omega)^{n}} \leqslant C\left(2 M_{1}+2 M_{2}\right)\|z\|_{V}\|w\|_{V} .
\end{aligned}
$$

Следовательно, $\|D(v)-D(u)\|_{V^{*}} \leqslant C_{1}\|v-u\|_{V}$. Таким образом, оператор $D: V \rightarrow V^{*}$ непрерывен. Последнее неравенство выполнено для почти всех $t \in(0, T)$, возведем его в квадрат и проинтегрируем по $t$ от 0 до $T$, получим

$$
\int_{0}^{T}\|D(v)-D(u)\|_{V^{*}}^{2} d x \leqslant C_{1} \int_{0}^{T}\|v-u\|_{V}^{2} d x .
$$

Так как $\|v-u\|_{V} \in L_{2}(0, T)$, то $\|D(v)-D(u)\|_{V^{*}} \in L_{2}(0, T)$ и, следовательно, $D(v)-D(u) \in$ $L_{2}\left(0, T ; V^{*}\right)$. Из последней оценки следует требуемое неравенство:

$$
\|D(v)-D(u)\|_{L_{2}\left(0, T ; V^{*}\right)} \leqslant C_{1}\|v-u\|_{L_{2}(0, T ; V)} .
$$


Покажем монотонность оператора $D(v)$. Здесь также применим теорему Лагранжа к той же функции, что и выше:

$$
\begin{gathered}
\langle D(v)-D(u), v-u\rangle= \\
=2 \int_{\Omega}\left[\phi\left(I_{2}(v)\right) \varepsilon(v)-\phi\left(I_{2}(u)\right) \varepsilon(u)\right]: \varepsilon(v-u) d x=2 \int_{\Omega} \frac{d}{d \delta}\left[\phi\left(I_{2}\left(v+\delta_{0} z\right)\right) \varepsilon\left(v+\delta_{0} z\right)\right]: \varepsilon(z) d x= \\
\quad=2 \int_{\Omega}\left[\phi\left(I_{2}\left(v+\delta_{0} z\right)\right) \varepsilon(z)+\frac{d}{d \delta} \phi\left(I_{2}\left(v+\delta_{0} z\right)\right) \varepsilon\left(v+\delta_{0} z\right)\right]: \varepsilon(z) d x= \\
\quad=2 \int_{\Omega}\left[\phi\left(I_{2}\left(v+\delta_{0} z\right)\right) \varepsilon(z): \varepsilon(z)+\frac{d}{d \delta} \phi\left(\left(\varepsilon\left(v+\delta_{0} z\right): \varepsilon\left(v+\delta_{0} z\right)\right)^{1 / 2}\right) \varepsilon\left(v+\delta_{0} z\right): \varepsilon(z)\right] d x= \\
=2 \int_{\Omega}\left[\phi\left(I_{2}\left(v+\delta_{0} z\right)\right) \varepsilon(z): \varepsilon(z)+\frac{\varepsilon\left(v+\delta_{0} z\right): \varepsilon(z)}{\left(\varepsilon\left(v+\delta_{0} z\right): \varepsilon\left(v+\delta_{0} z\right)\right)^{1 / 2}} \frac{d \phi\left(I_{2}\left(v+\delta_{0} z\right)\right)}{d I_{2}\left(v+\delta_{0} z\right)} \varepsilon\left(v+\delta_{0} z\right): \varepsilon(z)\right] d x= \\
\quad=2 \int_{\Omega}\left[\phi\left(I_{2}\left(v+\delta_{0} z\right)\right) \varepsilon(z): \varepsilon(z)+\frac{1}{I_{2}\left(v+\delta_{0} z\right)} \frac{d \phi\left(I_{2}\left(v+\delta_{0} z\right)\right)}{d I_{2}\left(v+\delta_{0} z\right)}\left(\varepsilon\left(v+\delta_{0} z\right): \varepsilon(z)\right)^{2}\right] d x .
\end{gathered}
$$

Если $d \phi(s) / d s \geqslant 0$, то подынтегральная функция больше либо равна нулю. Следовательно, $\langle D(u)-D(v), u-v\rangle \geqslant 0$.

Если $d \phi(s) / d s \leqslant 0$, то, используя неравенство $d \phi(s) / d s \geqslant-\phi(s) / s$, получим требуемое неравенство:

$$
\begin{gathered}
2 \int_{\Omega}\left[\phi\left(I_{2}\left(v+\delta_{0} z\right)\right) \varepsilon(z): \varepsilon(z)+\frac{1}{I_{2}\left(v+\delta_{0} z\right)} \frac{d \phi\left(I_{2}\left(v+\delta_{0} z\right)\right)}{d I_{2}\left(v+\delta_{0} z\right)}\left(\varepsilon\left(v+\delta_{0} z\right): \varepsilon(z)\right)^{2}\right] d x \geqslant \\
\geqslant 2 \int_{\Omega}\left[\phi\left(I_{2}\left(v+\delta_{0} z\right)\right) \varepsilon(z): \varepsilon(z)+\frac{I_{2}\left(v+\delta_{0} z\right)}{I_{2}^{2}\left(v+\delta_{0} z\right)} \frac{d \phi\left(I_{2}\left(v+\delta_{0} z\right)\right)}{d I_{2}\left(v+\delta_{0} z\right)} I_{2}^{2}\left(v+\delta_{0} z\right) I_{2}^{2}(z)\right] d x \geqslant \\
\geqslant 2 \int_{\Omega}\left[\phi\left(I_{2}\left(v+\delta_{0} z\right)\right) \varepsilon(z): \varepsilon(z)+I_{2}\left(v+\delta_{0} z\right) \frac{d \phi\left(I_{2}\left(v+\delta_{0} z\right)\right)}{d I_{2}\left(v+\delta_{0} z\right)} I_{2}^{2}(z)\right] d x \geqslant \\
\geqslant 2 \int_{\Omega}\left[\phi\left(I_{2}\left(v+\delta_{0} z\right)\right) I_{2}^{2}(z)-\phi\left(I_{2}\left(v+\delta_{0} z\right)\right) I_{2}^{2}(z)\right] d x \geqslant 0 .
\end{gathered}
$$

4. Аппроксимационная задача. Свойства операторов. В этом разделе рассматриваются свойства операторов, входящих в уравнение (3.1), а также оператора $K_{\varepsilon}(v)$, аппроксимирующего $K(v)$. Итак, введем оператор

$$
K_{\varepsilon}: V \rightarrow V^{*}, \quad\left\langle K_{\varepsilon}(v), \varphi\right\rangle=\int_{\Omega} \sum_{i, j=1}^{n} \frac{v_{i} v_{j}}{1+\varepsilon|v|^{2}} \frac{\partial \varphi_{j}}{\partial x_{i}} d x, \quad v \in V, \quad \varphi \in V ; \quad|v|=\sqrt{\sum_{i=1}^{n} v_{i} v_{i}} .
$$

Эта замена приводит к условию $v^{\prime}(t) \in L_{2}\left(0, T ; V^{*}\right)$ для решения соответствующего уравнения.

По аналогии определения слабого решения исходной задачи определим слабое решение аппроксимационной задачи. Рассмотрим следующую задачу, зависящую от параметра $\varepsilon>0$. Для заданных функций $f \in L_{2}\left(0, T ; V^{*}\right)$ и $v^{0} \in H$ найти функцию $v$, удовлетворяющую условиям

$$
v^{\prime}(t)+D(v)-K_{\varepsilon}(v)=f(t), \quad v(0)=v^{0} .
$$

Решение задачи (4.1) будем искать в банаховом пространстве

$$
W=\left\{v: v \in L_{2}(0, T ; V), v^{\prime} \in L_{2}\left(0, T ; V^{*}\right)\right\}, \quad\|v\|_{W}=\|v\|_{L_{2}(0, T ; V)}+\left\|v^{\prime}\right\|_{L_{2}\left(0, T ; V^{*}\right)} .
$$


Лемма 4.1. 1. Для любого $\varepsilon>0$ отображение $K_{\varepsilon}: L_{2}(0, T ; V) \rightarrow L_{2}\left(0, T ; V^{*}\right)$ корректно определено, непрерывно и справедлива оценка

$$
\left\|K_{\varepsilon}(v)\right\|_{L_{2}\left(0, T ; V^{*}\right)} \leqslant \frac{C_{1}}{\varepsilon}
$$

с некоторой константой $C_{1}$, не зависящей от $v$.

2. Для любого $\varepsilon>0$ отображение $K_{\varepsilon}: W \rightarrow L_{2}\left(0, T ; V^{*}\right)$ вполне непрерывно.

3. Для любого $\varepsilon \geqslant 0$ u $n \leqslant 4$ справедлива оченка

$$
\left\|K_{\varepsilon}(v)\right\|_{L_{1}\left(0, T ; V^{*}\right)} \leqslant C_{2}\|v\|_{L_{2}(0, T ; V)}^{2}
$$

с некоторой константой $C_{2}$, не зависящей от $v$ и $\varepsilon$.

Доказательство данной леммы приведено в [6, гл. 5, лемма 5.2].

Введем следующие обозначения:

$$
\begin{gathered}
\mathbf{L}: W \rightarrow L_{2}\left(0, T ; V^{*}\right) \times H, \quad \mathbf{L}(v)=\left(v^{\prime}+D(v),\left.v\right|_{t=0}\right) ; \\
\mathbf{K}_{\varepsilon}: W \subset L_{2}(0, T ; V) \rightarrow L_{2}\left(0, T ; V^{*}\right) \times H, \quad \mathbf{K}_{\varepsilon}(v)=\left(K_{\varepsilon}(v), 0\right) .
\end{gathered}
$$

Тогда задача (4.1) о существовании слабого решения аппроксимационной задачи эквивалентна операторному уравнению

$$
\mathbf{L}(v)=\mathbf{K}_{\varepsilon}(v)+\left(f, v_{0}\right) .
$$

Исследуем свойства оператора $\mathbf{L}$.

Лемма 4.2. Отображение $\mathbf{L}: W \rightarrow L_{2}\left(0, T ; V^{*}\right) \times H$ корректно определено, обратимо, причем для любых $v \in W$ и некоторой константы с справедлива оценка

$$
\|v\|_{W} \leqslant C_{3}\|\mathbf{L}(v)\|_{L_{2}\left(0, T ; V^{*}\right) \times H} .
$$

Oператор $\mathbf{L}^{-1}: L_{2}\left(0, T ; V^{*}\right) \times H \rightarrow W$ непрерывен $и$

$$
\left\|\mathbf{L}^{-1}\left(f, v^{0}\right)\right\|_{W} \leqslant C_{3}\left(\left\|v^{0}\right\|_{L_{2}(\Omega)^{n}}+\|f\|_{L_{2}\left(0, T ; V^{*}\right)}\right) .
$$

Доказательство. 1. Оператор взятия производной непрерывен, это следует из определения пространства $W$, оператор $D(v)$ непрерывен по доказанному выше. Так как вложение $W \subset$ $C([0, T], H)$ непрерывно (см. [12, гл. 3 , леммы $1.1,1.4])$, то оператор взятия следа функции $\left.v\right|_{t=0}$ корректно определен и непрерывен, а следовательно, корректно определен и непрерывен оператор L.

2. Докажем оценку (4.5). Для $v \in W$ обозначим $\mathbf{L}(v)=\left(\hat{f}, \hat{v}^{0}\right)$. При каждом фиксированном $t \in[0, T]$ применим функционалы $v^{\prime}+D(v)=\hat{f}$ к функции $v(t) \in V$

$$
\left\langle v^{\prime}(t), v(t)\right\rangle+\langle D(v), v(t)\rangle=\langle\hat{f}(t), v(t)\rangle .
$$

Так как

$$
\left\langle v^{\prime}(t), v(t)\right\rangle=\frac{1}{2} \frac{d}{d t}\|v(t)\|_{\left.L_{2}(\Omega)\right)^{n}}^{2}, \quad\langle\hat{f}(t), v(t)\rangle_{V} \leqslant\|\hat{f}(t)\|_{V^{*}}\|v(t)\|_{V},
$$

имеем в силу первого неравенства Корна (см. [13, ч. 1, п. 12])

$$
\langle D(v), v(t)\rangle=2 \int_{\Omega} \phi\left(I_{2}(v)\right) \varepsilon(v): \varepsilon(v) d x \geqslant 2 m_{1} c_{1} \int_{\Omega} \varepsilon(v): \varepsilon(v) d x \geqslant c_{2}\|v\|_{V}^{2} .
$$

Проинтегрируем полученное неравенство по переменной $t$ на отрезке $[0, t]$. Используя начальное условие для функции $u(t)$ и неравенство Коши

$$
a \cdot b \leqslant \frac{\varepsilon}{2} a^{2}+\frac{1}{2 \varepsilon} b^{2}, \quad \varepsilon, a, b>0,
$$

приходим к следующей оценке:

$$
\frac{1}{2}\|v(t)\|_{\left(L_{2}(\Omega)\right)^{n}}^{2}-\frac{1}{2}\left\|\hat{v}^{0}\right\|_{\left(L_{2}(\Omega)\right)^{n}}^{2}+c_{2} \int_{0}^{t}\|v(\tau)\|_{V}^{2} d \tau \leqslant \frac{1}{2 \varepsilon} \int_{0}^{t}\|\hat{f}(t)\|_{V^{*}}^{2} d \tau+\frac{\varepsilon}{2} \int_{0}^{t}\|v(\tau)\|_{V}^{2} d \tau .
$$


Теперь выбирая $\varepsilon=c_{2}$, получаем

$$
\frac{1}{2}\|v(t)\|_{\left(L_{2}(\Omega)\right)^{n}}^{2}+\frac{1}{2} c_{2} \int_{0}^{t}\|v(\tau)\|_{V}^{2} d \tau \leqslant \frac{1}{2}\left\|\hat{v}^{0}\right\|_{\left(L_{2}(\Omega)\right)^{n}}^{2}+\frac{1}{2 c_{2}} \int_{0}^{t}\|\hat{f}(t)\|_{V^{*}}^{2} d \tau .
$$

Умножид обе части неравенства на 2 , вычислим максимум по $t \in[0, T]$ :

$$
\max _{t \in[0, T]}\|v(t)\|_{\left(L_{2}(\Omega)\right)^{n}}^{2}+c_{2}\|v\|_{L_{2}(0, T ; V)}^{2} \leqslant\left\|\hat{v}^{0}\right\|_{\left(L_{2}(\Omega)\right)^{n}}^{2}+\frac{1}{c_{2}}\|\hat{f}\|_{L_{2}\left(0, T ; V^{*}\right)}^{2} .
$$

Используя неравенство $(a+b)^{2} \leqslant 2\left(a^{2}+b^{2}\right), a, b>0$, получаем итоговую оценку

$$
\max _{t \in[0, T]}\|v(t)\|_{\left(L_{2}(\Omega)\right)^{n}}+\|v\|_{L_{2}(0, T ; V)} \leqslant c_{3}\left(\left\|\hat{v}^{0}\right\|_{\left(L_{2}(\Omega)\right)^{n}}+\|\hat{f}\|_{L_{2}\left(0, T ; V^{*}\right)}\right)
$$

с некоторой константой $c_{3}$, зависящей от $m_{1}$. Для того чтобы оценить $\left\|v^{\prime}\right\|_{L_{2}\left(0, T ; V^{*}\right)}$, воспользуемся равенством $v^{\prime}=-D(v)+\hat{f}$, оценкой $\|D(v)\|_{L_{2}\left(0, T ; V^{*}\right)} \leqslant c_{4}\|v\|_{L_{2}(0, T ; V)}$ и полученной выше оценкой

$$
\begin{aligned}
\left\|v^{\prime}\right\|_{L_{2}\left(0, T ; V^{*}\right)} \leqslant\|\hat{f}\|_{L_{2}\left(0, T ; V^{*}\right)}+\|D(v)\|_{L_{2}\left(0, T ; V^{*}\right)} \leqslant\|\hat{f}\|_{L_{2}\left(0, T ; V^{*}\right)}+c_{4}\|v\|_{L_{2}(0, T ; V)} \leqslant \\
\leqslant\left(c_{3} c_{4}+1\right)\left(\left\|\hat{v}^{0}\right\|_{\left(L_{2}(\Omega)\right)^{n}}+\|\hat{f}\|_{L_{2}\left(0, T ; V^{*}\right)}\right)
\end{aligned}
$$

Таким образом, получаем требуемую оценку

$$
\|v\|_{W}=\|v\|_{L_{2}(0, T ; V)}+\left\|v^{\prime}\right\|_{L_{2}\left(0, T ; V^{*}\right)} \leqslant C_{3}\left(\left\|\hat{v}_{0}\right\|_{\left(L_{2}(\Omega)\right)^{n}}+\|\hat{f}\|_{L_{2}\left(0, T ; V^{*}\right)}\right)=C_{3}\|\mathbf{L}(v)\|_{L_{2}\left(0, T ; V^{*}\right) \times H}
$$

с некоторой константой $C_{3}$, зависящей от $m_{1}$.

3. Для доказательства обратимости отображения $\mathbf{L}$ достаточно применить теорему 1.1 из [3, гл. 4]. Так как оператор $D: V \rightarrow V^{*}$ непрерывен и монотонен, то все условия теоремы выполнены. Применение теоремы показывает, что для каждого $\left(\hat{f}, \hat{v}_{0}\right)$ существует решение $v \in L_{2}(0, T ; V)$, а следовательно, $v \in W$. Таким образом, оператор $\mathbf{L}$ обратим. Переписывая оценку (4.5) в виде

$$
\left\|\mathbf{L}^{-1}\left(\hat{f}, \hat{v}^{0}\right)\right\|_{W} \leqslant C_{3}\left(\left\|\hat{v}^{0}\right\|_{L_{2}(\Omega)^{n}}+\|\hat{f}\|_{L_{2}\left(0, T ; V^{*}\right)}\right),
$$

получаем, что оператор $\mathbf{L}^{-1}$ непрерывен.

5. Разрешимость аппроксимационных уравнений. Утверждение о разрешимости начальной задачи (4.1) для любых $\varepsilon>0$ устанавливается с помощью теории степени Лере-Шаудера.

Теорема 5.1. Пусть $\varepsilon>0$. Тогда для произвольных $f \in L_{2}\left(0, T ; V^{*}\right), v^{0} \in H$ задача (4.1) имеет по крайней мере одно решение $v \in W$.

Доказательство. Исследование задачи (4.1) заменим исследованием эквивалентного операторного уравнения

$$
\mathbf{L}(v)=\mathbf{K}_{\varepsilon}(v)+\left(f, v^{0}\right) .
$$

Применим оператор $\mathbf{L}^{-1}$ к обеим частям уравнения. Получим эквивалентное операторное уравнение

$$
v=\mathbf{L}^{-1}\left(\mathbf{K}_{\varepsilon}(v)+\left(f, v^{0}\right)\right)
$$

Обозначим через $k$ отображение, определяемое правой частью уравнения (5.1):

$$
k: W \rightarrow W, \quad v \rightarrow \mathbf{L}^{-1}\left(\mathbf{K}_{\varepsilon}(v)+\left(f, v^{0}\right)\right) .
$$

Тогда уравнение (5.1) можно записать в виде

$$
(I-k)(v)=0 .
$$

Отображение $k$ вполне непрерывно как композиция вполне непрерывного отображения $\left(\mathbf{K}_{\varepsilon}+\right.$ $\left.\left(f, v_{0}\right)\right)$ и непрерывного оператора $\mathbf{L}^{-1}$. Покажем, что степень Лере-Шаудера $\operatorname{deg}_{L S}\left(I-k, \bar{B}_{R}, 0\right)$ для отображения $(I-k)$ определена на шаре $\bar{B}_{R} \subset W$ достаточно большого радиуса $R$ и отлична от нуля.

Рассмотрим вспомогательное семейство операторных уравнений

$$
v=\lambda \mathbf{L}^{-1}\left(\mathbf{K}_{\varepsilon}+\left(f, v^{0}\right)\right), \quad \lambda \in[0,1] .
$$


Если $v \in W$ - решение одного из уравнений, то в силу оценок (4.5) и (4.2) имеем

$$
\begin{aligned}
& \|v\|_{W} \leqslant C_{3}\left\|\mathbf{K}_{\varepsilon}(v)+\left(f, v^{0}\right)\right\|_{L_{2}\left(0, T ; V^{*}\right) \times H} \leqslant \\
& \leqslant C_{3}\left(\left\|\mathbf{K}_{\varepsilon}(v)\right\|_{L_{2}\left(0, T ; V^{*}\right)}+\|f\|_{L_{2}\left(0, T ; V^{*}\right)}+\left\|v^{0}\right\|_{H}\right) \leqslant \\
& \quad \leqslant C_{3}\left(\frac{C_{1}}{\varepsilon}+\|f\|_{L_{2}\left(0, T ; V^{*}\right)}+\left\|v^{0}\right\|_{H}\right) .
\end{aligned}
$$

Выберем

$$
R>C_{3}\left(\frac{C_{1}}{\varepsilon}+\|f\|_{L_{2}\left(0, T ; V^{*}\right)}+\left\|v_{0}\right\|_{H}\right) ;
$$

тогда ни одно решение уравнений семейства (5.3) не принадлежит границе шара $B_{R} \subset W$. Поэтому отображение $I-\lambda k: W \times[0,1] \rightarrow W$ определяет гомотопию вполне непрерывных векторных полей на $B_{R}$. Следовательно, степень $\operatorname{deg}_{L S}\left(I-\lambda k, \bar{B}_{R}, 0\right)$ определена для каждого значения $\lambda \in[0,1]$, и в силу свойства гомотопической инвариантности степени имеем

$$
\operatorname{deg}_{L S}\left(I-k, \bar{B}_{R}, 0\right)=\operatorname{deg}_{L S}\left(I-\lambda k, \bar{B}_{R}, 0\right)=\operatorname{deg}_{L S}\left(I, \bar{B}_{R}, 0\right)=1,
$$

так как $0 \in B_{R}$. Отличие от нуля степени отображения обеспечивает существование решения операторного уравнения (5.2), а, следовательно, существование решения уравнения (4.4) и задачи (4.1).

\section{6. Априорная оценка решений и существование слабого решения.}

Теорема 6.1. Пусть $n \leqslant 4$. Для любого решения $v \in W$ задачи (4.1), существующего по теореме 5.1 с $\varepsilon>0$, справедливы оценки

$$
\begin{gathered}
\max _{t \in[0, T]}\|v(t)\|_{\left(L_{2}(\Omega)\right)^{n}}+\|v\|_{L_{2}(0, T ; V)} \leqslant C_{1}\left(\|f\|_{L_{2}\left(0, T ; V^{*}\right)}+\left\|v^{0}\right\|_{H}\right), \\
\left\|v^{\prime}\right\|_{L_{1}\left(0, T ; V^{*}\right)} \leqslant C_{2}\left(1+\|f\|_{L_{2}\left(0, T ; V^{*}\right)}+\left\|v^{0}\right\|_{H}\right)^{2},
\end{gathered}
$$

с константами $C_{1}, C_{2}$, не зависящими от $\varepsilon$.

Доказательство. Пусть $v \in W$-решение задачи (4.1), существующее по теореме 5.1 для некоторого $\varepsilon>0$. Повторяя рассуждения доказательства оценки (4.5) и используя тот факт, что $\left\langle K_{\varepsilon}(v(t)), v(t)\right\rangle=0$ для всех $t \in[0, T]$, отсюда нетрудно получить требуемую оценку:

$$
\max _{t \in[0, T]}\|v(t)\|_{\left(L_{2}(\Omega)\right)^{n}}+\|v\|_{L_{2}(0, T ; V)} \leqslant C_{1}\left(\|f\|_{L_{2}\left(0, T ; V^{*}\right)}+\left\|v^{0}\right\|_{H}\right) .
$$

Для того чтобы оценить $\left\|v^{\prime}\right\|_{L_{1}\left(0, T ; V^{*}\right)}$, воспользуемся равенством $v^{\prime}=-D(v)+K_{\varepsilon}(v)+f$. Отсюда

$$
\left\|v^{\prime}\right\|_{L_{1}\left(0, T ; V^{*}\right)} \leqslant\|D(v)\|_{L_{1}\left(0, T ; V^{*}\right)}+\left\|K_{\varepsilon}(v)\right\|_{L_{1}\left(0, T ; V^{*}\right)}+\|f\|_{L_{1}\left(0, T ; V^{*}\right)} .
$$

Используя непрерывность вложения $L_{2}\left(0, T ; V^{*}\right) \subset L_{1}\left(0, T ; V^{*}\right)$, с помощью неравенства Коши и оценки $\|D(v)\|_{L_{1}\left(0, T ; V^{*}\right)} \leqslant c_{1}\|v\|_{L_{2}(0, T ; V)}$ получим

$$
\|D(v)\|_{L_{1}\left(0, T ; V^{*}\right)} \leqslant c_{2}\|D(v)\|_{L_{2}\left(0, T ; V^{*}\right)} \leqslant c_{3}\|v\|_{L_{2}(0, T ; V)}, \quad\|f\|_{L_{1}\left(0, T ; V^{*}\right)} \leqslant \sqrt{T}\|f\|_{L_{2}\left(0, T ; V^{*}\right)} .
$$

Кроме того, для $\left\|K_{\varepsilon}(v)\right\|_{L_{1}\left(0, T ; V^{*}\right)}$ имеется оценку:

$$
\left\|K_{\varepsilon}(v)\right\|_{L_{1}\left(0, T ; V^{*}\right)} \leqslant c_{4}\|v\|_{L_{2}(0, T ; V)}^{2} .
$$

Подставляя полученные оценки в неравенство (6.3) и используя оценку (6.1), получим:

$$
\begin{aligned}
\left\|v^{\prime}\right\|_{L_{1}\left(0, T ; V^{*}\right)} \leqslant c_{3}\|v\|_{L_{2}(0, T ; V)}+c_{4}\|v\|_{L_{2}(0, T ; V)}^{2}+\sqrt{T}\|f\|_{L_{2}\left(0, T ; V^{*}\right)} & \leqslant \\
& \leqslant C_{2}\left(1+\|f\|_{L_{2}\left(0, T ; V^{*}\right)}+\left\|v_{0}\right\|_{H}\right)^{2} .
\end{aligned}
$$

Прежде чем переходить к доказательству теоремы о существовании слабых решений исходной задачи, сформулируем утверждение о предельном переходе для оператора $K_{\varepsilon}$. 
Лемма 6.1. GПредположим, что последовательность $\left\{v_{l}\right\}_{l=1}^{\infty}, v_{l} \in E$, удовлетворяет следующим условиям:

$$
\begin{aligned}
& v_{l} \rightarrow v^{*} \\
& v_{l} \rightarrow v^{*} \text { слабо в } E, \\
& v_{l} \rightarrow v^{*}
\end{aligned}
$$

Тогда

$$
K_{\varepsilon_{l}}\left(v_{l}\right) \rightarrow K\left(v^{*}\right) \quad \text { в смысле распределений при } l \rightarrow \infty, \quad \varepsilon_{l} \rightarrow 0 .
$$

Доказательство леммы 6.1 можно найти в [6, гл. 5, лемма 5.3].

Докажем теорему о существовании решения задачи (3.1), (3.2).

Теорема 6.2. Для каждой функции $f \in L_{2}\left(0, T ; V^{*}\right)$ u $v^{0} \in H$ задача (3.1), (3.2) имеет хотя бы одно решение $v \in W_{2,1}$.

Доказательство. Возьмем произвольную последовательность положительных чисел $\left\{\varepsilon_{l}\right\}_{l=1}^{\infty}$, $\varepsilon_{l} \rightarrow 0$. Для каждого $\varepsilon$ известно, что каждая соответствующая аппроксимационная задача имеет по крайней мере одно решение $v_{l} \in W$.

Из оценки (6.1) следует, что $\left\{v_{l}\right\}$ ограничена по норме $\|\cdot\|_{L_{2}(0, T ; V)}$ и $\|\cdot\|_{L_{\infty}(0, T, H)}$, а из оценки (6.2) последовательность $\left\{v_{l}^{\prime}\right\}$ ограничена по норме пространства $L_{1}\left(0, T ; V^{*}\right)$. Тогда, не уменьшая общности рассуждений, будем полагать что

$$
\begin{array}{llll}
v_{l} \rightarrow v^{*} & \text { слабо в } E, & v_{l} \rightarrow & *_{\text {- слабо в } L_{\infty}(0, T ; H),} \\
v_{l} \rightarrow v^{*} & \text { сильно в } L_{2}\left(Q_{T}\right)^{n}, & v_{l} \rightarrow v^{*} & \text { почти всюду } Q_{T}, \\
v_{l}^{\prime} \rightarrow v^{\prime *} & \text { в смысле распределений. } & &
\end{array}
$$

Так как оператор $D$ слабо непрерывен, то будем полагать, что $D\left(v_{l}\right) \rightarrow D\left(v^{*}\right)$ слабо в $E^{*}$, а следовательно в смысле распределений со значениями в $V^{*}$.

В силу леммы (6.1) выполнена следующая сходимость:

$$
K_{\varepsilon_{l}}\left(v_{l}\right) \rightarrow K\left(v^{*}\right) \text { в смысле распределений. }
$$

Для завершения доказательства осталось перейти к пределу в смысле распределений при $l \rightarrow \infty$ в равенстве

Получим равенство

$$
v_{l}^{\prime}+D\left(v_{l}\right)-K_{\varepsilon_{l}}\left(v_{l}\right)=f .
$$

$$
v^{\prime *}+D\left(v^{*}\right)-K\left(v^{*}\right)=f .
$$

Следовательно, $v^{*}$ - слабое решение задачи (4.1). Заметим, что так как $v^{*} \in L_{2}(0, T ; V) \cap$ $L_{\infty}(0, T ; H)$, то из равенства (4.1) следует, что $v^{\prime *} \in L_{1}\left(0, T ; V^{*}\right)$.

7. Оценки решений. В этом пункте исследуются оценки решений системы нелинейно-вязкой жидкости. Данные результаты необходимы нам для доказательства существования аттракторов.

Замечание 7.1. Далее будем предполагать, что $f \in V^{*}$.

Лемма 7.1. Пусть $f \in V^{*}, v^{0} \in H$. Тогда на отрезке $[0, T]$ существует решение $v(t)$ задачи (3.1), (3.2), удовлетворяющее неравенству

$$
\frac{1}{2}\|v(t)\|_{L_{\infty}(t, t+1 ; H)}^{2}+\frac{C_{1}}{4}\|v(t)\|_{L_{2}(t, t+1 ; V)}^{2} \leqslant e^{-2 t}\|v\|_{L_{\infty}\left(\mathbb{R}_{+} ; H\right)}^{2}+\frac{3}{2 C_{1}}\|f\|_{V^{*}}^{2} .
$$

Доказательство. Доказательство проведем в два этапа. На первом этапе докажем, что данной оценке удовлетворяет слабое решение аппроксимационной задачи. На втором этапе при помощи предельного перехода распространим эту оценку на решения задачи (3.1), (3.2). Сначала получим оценку первого слагаемого в левой части равенства (7.1). Для доказательства воспользуемся оценкой

$$
\frac{1}{2} \frac{d}{d t}\|v(t)\|_{H}^{2}+c_{1}\|v(t)\|_{V}^{2} \leqslant\|f\|_{V^{*}}\|v(t)\|_{V}
$$


и положим $\bar{v}(t)=e^{\gamma t} v(t)$, где $v(t)$ - решение аппроксимационной задачи на $[0, T]$, а $\gamma$ - некоторое число больше нуля. Подставив новые функции в неравенство, получим

$$
\frac{1}{2} \frac{d}{d t} e^{-2 \gamma t}\|\bar{v}(t)\|_{H}^{2}+c_{1} e^{-2 \gamma t}\|\bar{v}(t)\|_{V}^{2} \leqslant e^{-\gamma t}\|f\|_{V^{*}}\|\bar{v}(t)\|_{V} .
$$

Раскрыв производную произведения и умножив обе части на $e^{2 \gamma t}$, получаем

$$
\frac{1}{2} \frac{d}{d t}\|\bar{v}(t)\|_{H}^{2}-\gamma\|\bar{v}(t)\|_{H}^{2}+c_{1}\|\bar{v}(t)\|_{V}^{2} \leqslant e^{\gamma t}\|f\|_{V^{*}}\|\bar{v}(t)\|_{V}
$$

Использовав следствие из неравенства Пуанкаре $\|v\|_{H} \leqslant k\|v\|_{V}(\forall v \in V)$, придем к следующему неравенству:

$$
\frac{1}{2} \frac{d}{d t}\|\bar{v}(t)\|_{H}^{2}-\gamma k^{2}\|v\|_{V}^{2}+c_{1}\|\bar{v}(t)\|_{V}^{2} \leqslant e^{\gamma t}\|f\|_{V^{*}}\|\bar{v}(t)\|_{V}
$$

Положим $\gamma=c_{1} /\left(2 k^{2}\right)$. Тогда придем к оценке

$$
\frac{1}{2} \frac{d}{d t}\|\bar{v}(t)\|_{H}^{2}+\frac{c_{1}}{2}\|\bar{v}(t)\|_{V}^{2} \leqslant e^{\gamma t}\|f\|_{V^{*}}\|\bar{v}(t)\|_{V}
$$

Используя неравенство Коши (4.7) с константой $\alpha=1 / c_{1}$, получим

$$
\frac{1}{2} \frac{d}{d t}\|\bar{v}(t)\|_{H}^{2}+\frac{c_{1}}{2}\|\bar{v}(t)\|_{V}^{2} \leqslant \frac{e^{2 \gamma t}}{2 c_{1}}\|f\|_{V^{*}}^{2}+\frac{c_{1}}{2}\|\bar{v}(t)\|_{V}^{2},
$$

или

$$
\frac{d}{d t}\|\bar{v}(t)\|_{H}^{2} \leqslant \frac{e^{2 \gamma t}}{2 c_{1}}\|f\|_{V^{*}}^{2} .
$$

Заменив $t$ на $s$ и интегрируя последнее неравенство от 0 до $t$, находим

$$
\|\bar{v}(t)\|_{H}^{2} \leqslant\left\|v^{0}\right\|_{H}^{2}+\frac{1}{2 c_{1}} \int_{0}^{t} e^{2 \gamma s}\|f\|_{V^{*}}^{2} d s=\left\|v^{0}\right\|_{H}^{2}+\frac{1}{2 c_{1}}\|f\|_{V^{*}}^{2}\left(e^{2 \gamma t}-1\right) .
$$

Возвратившись к исходным функциям и умножив обе части равенства на $e^{-2 \gamma t}$, получим

$$
\|v(t)\|_{H}^{2} \leqslant e^{-2 \gamma t}\left\|v^{0}\right\|_{H}^{2}+\frac{1}{2 c_{1}}\|f\|_{V^{*}}^{2}\left(1-e^{-2 \gamma t}\right),
$$

или

$$
\|v(t)\|_{H}^{2} \leqslant e^{-2 \gamma t}\left\|v^{0}\right\|_{H}^{2}+\frac{1}{2 c_{1}}\|f\|_{V^{*}}^{2}
$$

Функция $\|v(t)\|_{H}^{2}$ непрерывна на $[0, T]$, поэтому последнее неравенство выполняется всюду на $[0, T]$, и можно перейти к максимуму по $[t, t+1] \subset[0, T-1]$. Получаем оценку

$$
\max _{s \in[t, t-1]}\left(\|v(s)\|_{H}^{2}\right) \leqslant e^{-2 \gamma t}\left\|v^{0}\right\|_{H}^{2}+\frac{1}{2 c_{1}}\|f\|_{V^{*}}^{2} .
$$

Чтобы получить оценку второго слагаемого в левой части неравенства (7.1), применим неравенство Коши с константой $\alpha=c_{1} / 2$ к правой части неравенства (7.2):

$$
\frac{1}{2} \frac{d}{d t}\|v(t)\|_{H}^{2}+\frac{c_{1}}{2}\|v(t)\|_{V}^{2} \leqslant \frac{1}{c_{1}}\|f\|_{V^{*}}^{2}+\frac{c_{1}}{4}\|v(t)\|_{V}^{2},
$$

или

$$
\frac{1}{2} \frac{d}{d t}\|v(t)\|_{H}^{2}+\frac{c_{1}}{4}\|v(t)\|_{V}^{2} \leqslant \frac{1}{c_{1}}\|f\|_{V^{*}}^{2} .
$$

Заменяя $t$ на $s$ и интегрируя по отрезку $[t, t+1] \subset[0, T-1]$, получаем

$$
\frac{1}{2}\|v(t+1)\|_{H}^{2}-\frac{1}{2}\|v(t)\|_{H}^{2}+\frac{c_{1}}{4} \int_{t}^{t+1}\|v(s)\|_{V}^{2} d s \leqslant \frac{1}{c_{1}}\|f\|_{V^{*}}^{2} .
$$


В силу непрерывности функции $v$ на $[0, T]$ со значениями в $H$ последнее равенство выполняется всюду на $[0, T-1]$. Складывая его с равенством (7.4), получаем

$$
\frac{1}{2}\|v(t+1)\|_{H}^{2}-\frac{1}{2}\|v(t)\|_{H}^{2}+\max _{s \in[t, t-1]}\left(\|v(s)\|_{H}^{2}\right)+\frac{c_{1}}{4} \int_{t}^{t+1}\|v(s)\|_{V}^{2} d s \leqslant e^{-2 \gamma t}\left\|v_{\varepsilon}^{0}\right\|_{H}^{2}+\left(\frac{1}{2 c_{1}}+\frac{1}{c_{1}}\right)\|f\|_{V^{*}}^{2} .
$$

Удалим положительные слагаемые в левой части этого равенства, воспользовавшись тем, что

$$
\max _{s \in[t, t-1]}\left(\frac{1}{2}\|v(s)\|_{H}^{2}\right)-\frac{1}{2}\|v(t)\|_{H}^{2} \geqslant 0 .
$$

Тогда

$$
\frac{1}{2} \max _{s \in[t, t-1]}\left(\|v(s)\|_{H}^{2}\right)+\frac{c_{1}}{4} \int_{t}^{t+1}\|v(s)\|_{V}^{2} d s \leqslant e^{-2 \gamma t}\left\|v^{0}\right\|_{H}^{2}+\frac{3}{2 c_{1}}\|f\|_{V^{*}}^{2} .
$$

Последнее неравенство представляет собой оценку (7.1) для аппроксимационной задачи.

Доказательства существования решений системы (3.1), (3.2), удовлетворяющих оценке (7.1), осуществим с помощью предельного перехода. Пусть $\left\{\varepsilon_{l}\right\}_{l=1}^{\infty}$ - сходящаяся к нулю последовательность, такая, что $\varepsilon_{l}>0$, и пусть $v_{l}$-решение на отрезке $[0, T]$ аппроксимационной задачи с $\varepsilon=\varepsilon_{l}$. Без ограничения общности можно считать, что существует предел

$$
v=\varliminf_{l \rightarrow \infty} v_{l},
$$

который подразумевается в смысле слабой сходимости в $L_{2}(0, T ; V)$ и $*$-слабой сходимости в $L_{\infty}(0, T ; H)$. Эти пределы также имеют место в смысле слабой сходимости в $L_{2}(t, t+1 ; V)$ и $*$-слабой сходимости в $L_{\infty}(t, t+1 ; H)$ для любого $t \geqslant 0$. Предельная функция является решением задачи (3.1), (3.2).

Также в силу слабой сходимости выполнены соотношения

$$
\|v\|_{L_{2}(t, t+1 ; V)} \leqslant \varliminf_{l \rightarrow \infty}\left\|v_{l}\right\|_{L_{2}(t, t+1 ; V)}, \quad\|v\|_{L_{\infty}(t, t+1 ; H)} \leqslant \underline{l \rightarrow \infty}\left\|v_{l}\right\|_{L_{\infty}(t, t+1 ; H)} .
$$

Следовательно,

$$
\begin{aligned}
& \frac{1}{2}\|v\|_{L_{\infty}(t, t+1 ; H)}^{2}+\frac{c_{1}}{4}\|v\|_{L_{2}(t, t+1 ; V)}^{2} \leqslant \varliminf_{l \rightarrow \infty} \frac{1}{2}\left\|v_{l}\right\|_{L_{\infty}(t, t+1 ; H)}^{2}+\varliminf_{l \rightarrow \infty} \frac{c_{1}}{4}\left\|v_{l}\right\|_{L_{2}(t, t+1 ; V)}^{2} \leqslant \\
& \leqslant \varliminf_{l \rightarrow \infty}\left(\frac{1}{2}\left\|v_{l}\right\|_{L_{\infty}(t, t+1 ; H)}^{2}+\frac{c_{1}}{4}\left\|v_{l}\right\|_{L_{2}(t, t+1 ; V)}^{2}\right) \leqslant e^{-2 \gamma t}\left\|v^{0}\right\|_{H}^{2}+\frac{3}{2 c_{1}}\|f\|_{V^{*}}^{2} .
\end{aligned}
$$

Осталось отметить, что $\left\|v^{0}\right\|_{H}=\|v(0)\|_{H} \leqslant\|v\|_{L_{\infty}\left(\mathbb{R}^{+} ; H\right)}$. Таким образом, получили в точности оценку (7.1).

Лемма 7.2. Пусть $v$-решение задачи (3.1), (3.2) на $[0, T]$. Тогда $v^{\prime} \in L_{4 / 3}\left(0, T ; V^{*}\right)$, и при $0 \leqslant t \leqslant T-1$ имеет место оценка

$$
\left\|v^{\prime}\right\|_{L_{4 / 3}\left(t, t+1 ; V^{*}\right)} \leqslant C_{2}\left(\|v\|_{L_{\infty}(t, t+1 ; H)}^{1 / 2}\|v\|_{L_{2}(t, t+1 ; V)}^{3 / 2}+\|v\|_{L_{\infty}(t, t+1 ; V)}+\|f\|_{V^{*}}\right) .
$$

с постоянной $C_{2}$, не зависящей от $v$.

Доказательство. При всех $\varphi \in V$ имеем равенство

$$
\left\langle v^{\prime}, \varphi\right\rangle=-\langle D(v), \varphi\rangle+\langle K(v), \varphi\rangle+\langle f(t), \varphi\rangle .
$$

Данное тождество выполняется почти всюду на $[0, T]$. Покажем, что слагаемые в правой части принадлежат пространству $L_{4 / 3}(0, T)$ и оценим их нормы в пространствах $L_{4 / 3}(t, t+1), t \leqslant T-1$. Так как при почти всех $s \in[0, T]$ имеем $v(t) \in V$, и имеет место вложение $V \subset\left(L_{4}(\Omega)\right)^{n}$, то 
$v_{j}(t) v(t) \in\left(L_{4}(\Omega)\right)^{n}$. С помощью интегрального и арифметического неравенства Коши-Буняковского получаем

$$
\begin{aligned}
&\left|\sum_{i=1}^{n}\left(v_{j}(t) v(t), \frac{\partial \varphi}{\partial x_{i}}\right)\right| \leqslant \sum_{i=1}^{n}\left\|v_{j}(t) v(t)\right\|_{\left(L_{2}(\Omega)\right)^{n}}\left\|\frac{\partial \varphi}{\partial x_{i}}\right\|_{\left(L_{2}(\Omega)\right)^{n}} \leqslant \\
& \leqslant\left(\sum_{i=1}^{n}\left\|v_{i}(t) v(t)\right\|_{\left(L_{2}(\Omega)\right)^{n}}^{2}\right)^{1 / 2}\left(\sum_{i=1}^{n}\left\|\frac{\partial \varphi}{\partial x_{i}}\right\|_{\left(L_{2}(\Omega)\right)^{n}}^{2}\right)^{1 / 2} .
\end{aligned}
$$

Второй множитель полученного выражения равен $\|\varphi\|_{V}$ :

$$
\left(\sum_{i=1}^{n}\left\|\frac{\partial \varphi}{\partial x_{i}}\right\|_{\left(L_{2}(\Omega)\right)^{n}}^{2}\right)^{1 / 2}=\left(\sum_{i, j=1}^{n}\left\|\frac{\partial \varphi_{j}}{\partial x_{i}}\right\|_{\left(L_{2}(\Omega)\right)^{n}}^{2}\right)^{1 / 2}=\|\varphi\|_{V} .
$$

Оценим первый множитель с помощью неравенства Гёльдера:

$$
\begin{aligned}
& \sum_{i=1}^{n}\left\|v_{j}(t) v(t)\right\|_{\left(L_{2}(\Omega)\right)^{n}}^{2}=\sum_{i, j=1}^{n}\left\|v_{i}(t) v_{j}(t)\right\|_{L_{2}(\Omega)}^{2} \leqslant \\
& \leqslant \sum_{i, j=1}^{n}\left\|v_{i}(t)\right\|_{L_{4}(\Omega)}^{2}\left\|v_{j}(t)\right\|_{L_{4}(\Omega)}^{2}=\left(\sum_{i=1}^{n}\left\|v_{i}(t)\right\|_{L_{4}(\Omega)}^{2}\right)\left(\sum_{j=1}^{n}\left\|v_{j}(t)\right\|_{L_{4}(\Omega)}^{2}\right)=\|v\|_{\left(L_{4}(\Omega)\right)^{n}}^{4} .
\end{aligned}
$$

Таким образом,

$$
\left|\sum_{i=1}^{n}\left(v_{j}(t) v(t), \frac{\partial \varphi}{\partial x_{i}}\right)\right| \leqslant\|v\|_{\left(L_{4}(\Omega)\right)^{n}}^{2}\|\varphi\|_{V}
$$

Из полученной оценки и неравенства

$$
\|z\|_{\left(L_{4}(\Omega)\right)^{n}} \leqslant K\|z\|_{\left(L_{2}(\Omega)\right)^{n}}^{1 / 4}\|\nabla z\|_{\left(L_{2}(\Omega)\right)^{n}}^{3 / 4} \quad(n=2,3),
$$

выполняющегося для функций $z \in H_{0}^{1}$ с константой $K$, не зависящей от $z$, получаем следующее неравенство:

$$
\left|\sum_{i=1}^{n}\left(v_{j}(t) v(t), \frac{\partial \varphi}{\partial x_{i}}\right)\right| \leqslant K^{2}\|v(t)\|_{H}^{1 / 2}\|v(t)\|_{V}^{3 / 2}\|\varphi\|_{V} .
$$

Для первого и третьего слагаемых в правой части тождества (7.5) имеем соотношения

$$
|\langle D(v), \varphi\rangle| \leqslant c_{1}\|v\|_{V}\|\varphi\|_{V} ; \quad|\langle f, \varphi\rangle| \leqslant\|f\|_{V^{*}}\|\varphi\|_{V}
$$

Складывая полученные неравенства, получаем оценку

$$
\left|\left\langle v^{\prime}, \varphi\right\rangle\right| \leqslant\left(K^{2}\|v(t)\|_{H}^{1 / 2}\|v(t)\|_{V}^{3 / 2}+c_{1}\|v(t)\|_{V}+\|f\|_{V^{*}}\right)\|\varphi\|_{V},
$$

откуда в силу произвольности $\varphi$ следует

$$
\left\|v^{\prime}\right\|_{V^{*}} \leqslant K^{2}\|v(t)\|_{H}^{1 / 2}|v(t)|_{V}^{3 / 2}+c_{1}\|v(t)\|_{V}+\|f\|_{V^{*}} .
$$

Так как функция $v$ принадлежит классу $L_{2}(0, T ; V) \cap L_{\infty}(0, T ; H)$, то каждое слагаемое в правой части неравенства суммируемо на $[0, T]$ со степенью $4 / 3$, а значит левая часть неравенства тоже суммируема с этой степенью. Далее, при $t \leqslant T-1$ с помощью неравенства Минковского получаем

$$
\begin{aligned}
\left\|v^{\prime}\right\|_{L_{4 / 3}\left(t, t+1 ; V^{*}\right)} & =\left(\int_{t}^{t+1}\left\|v^{\prime}\right\|_{V^{*}}^{43} d s\right)^{3 / 4} \leqslant\left(\int_{t}^{t+1} K^{8 / 3}\|v\|_{H}^{2 / 3}\|v\|_{V}^{2} d s\right)^{3 / 4}+\left(\int_{t}^{t+1}\|v\|_{V}^{4 / 3} d s\right)^{3 / 4}+ \\
& +\left(\int_{t}^{t+1}\|f\|_{V^{*}}^{4 / 3} d s\right)^{3 / 4} \leqslant K^{2}\|v\|_{L_{\infty}(t, t+1 ; H)}^{12}\|v\|_{L_{2}(t, t+1 ; V)}^{3 / 2}+\|v\|_{L_{\infty}(t, t+1 ; V)}+\|f\|_{V^{*}} .
\end{aligned}
$$


В итоге получим требуемую оценку

$$
\left\|v^{\prime}\right\|_{L_{4 / 3}\left(t, t+1 ; V^{*}\right)} \leqslant C_{2}\left(\|v\|_{L_{\infty}(t, t+1 ; H)}^{1 / 2}\|v\|_{L_{2}(t, t+1 ; V)}^{3 / 2}+\|v\|_{L_{\infty}(t, t+1 ; V)}+\|f\|_{V^{*}}\right)
$$

с некоторой константой $C_{2}$, не зависящей от $v$.

Следствие 7.1. Существует такая постоянная $R_{0}$, что для любого решения $v$ системъ (3.1), (3.2) на $[0, T]$, удовлетворяющая оценке (7.1), выполняется неравенство

$$
\|v\|_{L_{\infty}(t, t+1 ; H)}+\|v\|_{L_{2}(t, t+1 ; V)}+\left\|v^{\prime}\right\|_{L_{4 / 3}\left(t, t+1 ; V^{*}\right)} \leqslant R_{0}\left(1+e^{-2 \gamma t}\|v\|_{L_{\infty}\left(\mathbb{R}_{+} ; H\right)}^{2}\right) .
$$

8. Траекторный и глобальный аттракторы системы нелинейно-вязкой жидкости. Для введения пространства траекторий системы нелинейно-вязкой жидкости будем использовать следующую конкретизацию банаховых пространств $E=H$ и $E_{0}=V^{*}$.

В качестве пространства траекторий рассмотрим множество $\mathcal{H}^{+}$, состоящее из функций $v$, которые принадлежат классу

$$
v \in L_{2}^{\text {loc }}\left(\mathbb{R}_{+}, V\right) \cap L_{\infty}\left(\mathbb{R}_{+}, H\right),
$$

для всех $\varphi \in V$ и для почти всех значений $t \in \mathbb{R}_{+}$удовлетворяют интегральному тождеству

$$
\left\langle v^{\prime}, \varphi\right\rangle+2 \int_{\Omega} \phi\left(I_{2}(v)\right) \varepsilon(v): \varepsilon(\varphi) d x-\int_{\Omega} \sum_{i, j=1}^{n} v_{i} v_{j} \frac{\partial \varphi_{j}}{\partial x_{i}} d x=\langle f, \varphi\rangle,
$$

а также энергетической оценке

$$
\frac{1}{2}\|v(t)\|_{L_{\infty}(t, t+1 ; H)}^{2}+\frac{C_{1}}{4}\|v(t)\|_{L_{2}(t, t+1 ; V)}^{2} \leqslant e^{-2 \gamma t}\|v\|_{L_{\infty}\left(\mathbb{R}^{+} ; H\right)}^{2}+\frac{3}{2 C_{1}}\|f\|_{V^{*}}^{2} .
$$

Замечание 8.1. Если функция $v$ удовлетворяет введенному выше пространству траекторий, то функция $v$ имеет производную $v^{\prime} \in L_{4 / 3}^{l o c}\left(\mathbb{R}_{+}, V^{*}\right)$, что следует из леммы (7.2).

Чтобы данное определение пространства траекторий было корректно, нужно убедиться, что пространство непусто, и проверить включение

$$
\mathcal{H}^{+} \subset C\left(\mathbb{R}_{+} ; V^{*}\right) \cap L_{\infty}\left(\mathbb{R}_{+} ; H\right) .
$$

Включение $\mathcal{H}^{+} \subset L_{\infty}\left(\mathbb{R}_{+} ; H\right)$ непосредственно следует из определения пространства траекторий. Покажем непрерывность траекторий. Если $v$-некоторая траектория, то согласно замечанию (8.1) на произвольном отрезке $[0, M]$ имеем $\Pi_{M} v \in L_{\infty}(0, M ; H), \Pi_{M} v^{\prime} \in L_{4 / 3}\left(0, M ; V^{*}\right)$, поэтому для тройки пространств $H \subset H^{*} \subset V^{*}$ следует, что $\Pi_{M} v \in C C\left(0, M ; V^{*}\right)$ (см. [17]). Так как это верно при любом $M$, следовательно, $v \in C\left(\mathbb{R}_{+} ; V^{*}\right)$, что и требовалось.

Теорема 8.1. Пусть $f \in V^{*}$. Тогда для любого $v^{0} \in H$ существует траектория $v \in \mathcal{H}^{+}$, удовлетворяющая начальному условию $v(0)=v^{0}$.

Доказательство. Пусть $v_{m}$ - решение задача $(2.4)$ с начальным условием $v_{m}(0)=v_{m}^{0}$ на $\left[0, T_{m}\right]$, где $\left\{T_{m}\right\}$ - возрастающая, стремящаяся к бесконечности последовательность положительных чисел. Отметим, что решение $v_{m}$ на любом отрезке $\left[0, T_{m}\right]$ удовлетворяет оценке $(7.1)$, и, следовательно, оценке (7.6). Продолжим эту функцию константой по непрерывности на $\mathbb{R}_{+}$до функции $\tilde{v}_{m}$

$$
\tilde{v}_{m}= \begin{cases}v_{m}(t), & \text { если } 0<t<T_{m} ; \\ v_{m}\left(T_{m}\right), & \text { если } T_{m}<t .\end{cases}
$$

Из неравенства (7.6) следует, что последовательность $\tilde{v}_{m}$ ограничена в $L_{\infty}\left(\mathbb{R}_{+} ; H\right)$. Поэтому без ограничения общности можно считать, что она сходится к некоторой функции $v^{*} \in L_{\infty}\left(\mathbb{R}_{+} ; H\right)$ *-слабо в $L_{\infty}\left(\mathbb{R}_{+} ; H\right)$.

Докажем, что $v^{*}$ - решение системы (2.4), удовлетворяющее неравенству (8.2) при всех $t \in \mathbb{R}_{+}$ и, следовательно, это искомая траектория. Покажем, что можно совершить предельный переход 
в равенстве (2.4) и в неравенстве (8.2). Пусть $M$ некоторое положительное число. При всех достаточно больших $m$ функция $\Pi_{M} \tilde{v}_{m}$ является решением на $[0, M]$. Т.е. удовлетворяет уравнению

$$
\left\langle\tilde{v}_{m}^{\prime}, \varphi\right\rangle=-\left\langle D\left(\tilde{v}_{m}\right), \varphi\right\rangle+\left\langle K\left(\tilde{v}_{m}\right), \varphi\right\rangle+\langle f(t), \varphi\rangle
$$

для всех $\varphi \in V$ почти всюду на $[0, M]$. Кроме того, функция $v^{*}$ удовлетворяет при $t \in[0, M-1]$ неравенству

$$
\frac{1}{2}\left\|v_{m}\right\|_{L_{\infty}(t, t+1 ; H)}^{2}+\frac{c_{1}}{4}\left\|v_{m}\right\|_{L_{2}(t, t+1 ; V)}^{2} \leqslant e^{-2 \gamma t}\left\|v^{0}\right\|_{H}^{2}+\frac{3}{2 c_{1}}\|f\|_{V^{*}}^{2}
$$

а значит, согласно лемме (7.6), и неравенству

$$
\left\|v_{m}\right\|_{L_{\infty}(t, t+1 ; H)}+\left\|v_{m}\right\|_{L_{2}(t, t+1 ; V)}+\left\|v_{m}^{\prime}\right\|_{L_{4 / 3}\left(t, t+1 ; V^{*}\right)} \leqslant R_{0}\left(1+e^{-2 \gamma t}\left\|v^{0}\right\|_{L_{\infty}\left(R_{+} ; H\right)}^{2}\right) .
$$

Отсюда следует ограниченность последовательности $\left\{\Pi_{M} \tilde{v}_{m}\right\}$ в нормах пространств $L_{\infty}(0, M ; H)$ и $L_{2}(0, M ; V)$, а также в норме пространства $L_{4 / 3}\left(t, t+1 ; V^{*}\right)$. Отсюда следует, что последовательность $\left\{\Pi_{M} \tilde{v}_{m}\right\}$ сходится к предельной функции $\left\{\Pi_{M} v^{*}\right\}$ не только $*$-слабо в пространстве $L_{\infty}(0, M ; H)$, но и слабо в пространстве $L_{2}(0, M ; V)$, а с учетом ограниченности производных также сильно в $L_{2}(0, M ; H)$. Теперь можно перейти к пределу в равенстве $(8.3)$ и сделать вывод о том, что $v^{*}$ является решением системы $(2.4)$.

Остается убедиться, что можно перейти к пределу в оценке (8.4). Отметим, что правая часть не зависит от $m$. Из того, что последовательность $\tilde{v}_{m}$ сходится к $v^{*} *$-слабо в $L_{\infty}(0, M ; H)$ следует, что она сходится и $*$-слабо в $L_{\infty}(t, t+1 ; H), t \in[0, M-1]$, а потому

$$
\left\|v^{*}\right\|_{L_{\infty}(t, t+1 ; H)} \leqslant \underline{\lim _{m \rightarrow \infty}}\left\|\tilde{v}_{m}\right\|_{L_{\infty}(t, t+1 ; H)} .
$$

Аналогичным образом доказывается неравенство

$$
\left\|v^{*}\right\|_{L_{\infty}(t, t+1 ; V)} \leqslant \varliminf_{m \rightarrow \infty}\left\|\tilde{v}_{m}\right\|_{L_{\infty}(t, t+1 ; V)}
$$

Эти неравенства показывают, что в (8.4) можно перейти к пределу с сохранением знака неравенства. В силу произвольности $M$ получаем требуемое.

Следствие 8.1. Для любой траектории $v \in \mathcal{H}^{+}$выполняется неравенство

$$
\|v\|_{L_{\infty}(t, t+1 ; H)}+\|v\|_{L_{2}(t, t+1 ; V)}+\left\|v^{\prime}\right\|_{L_{4 / 3}\left(t, t+1 ; V^{*}\right)} \leqslant R_{0}\left(1+e^{-2 \gamma t}\|v\|_{L_{\infty}\left(R_{+} ; H\right)}^{2}\right)
$$

при $t \in \mathbb{R}_{+}$, где $R^{0}$ - константа из леммы 7.6 .

Теорема 8.2. Пусть $f \in V^{*}$. Тогда существует минимальный траекторный аттрактор $\mathcal{U}$ пространства траекторий $\mathcal{H}^{+}$.

Доказательство. Рассмотрим множество $P \subset C\left(\mathbb{R}_{+} ; V^{*}\right) \cap L_{\infty}\left(\mathbb{R}_{+} ; H\right)$, состоящее из таких траекторий $v$, что при всех $t \geqslant 0$ удовлетворяют неравенству

$$
\|v\|_{L_{\infty}(t, t+1 ; H)}+\|v\|_{L_{2}(t, t+1 ; V)}+\left\|v^{\prime}\right\|_{L_{4 / 3}\left(t, t+1 ; V^{*}\right)} \leqslant 2 R_{0}
$$

где $R_{0}$ - константа из леммы 7.6. Очевидно, множество $P$ ограничено в $L_{\infty}\left(\mathbb{R}_{+} ; H\right)$. Покажем, что оно относительно компактно в $C\left(\mathbb{R}_{+}, V^{*}\right)$. Докажем, что при любом $M>0$ множество $\Pi_{M} P$ относительно компактно в $C\left(0, M ; V^{*}\right)$. Однако это следует из того, что сами траектории $v \in P$ ограничены в $L_{\infty}(0, M ; H)$, а производные $v^{\prime}$ ограничены в $L_{4 / 3}\left(t, t+1 ; V^{*}\right)$.

Покажем, что множество $P$ замкнуто в $C\left(\mathbb{R}_{+}, V^{*}\right)$ и, следовательно, компактно в этом пространстве. Пусть последовательность $\left\{v_{m}\right\} \subset P$ сходится к $v$ в метрике пространства $C\left(\mathbb{R}_{+}, V^{*}\right)$. Покажем, что $v \in P$.

Из определения множества $P$ следует выполнение неравенства

$$
\left\|v_{m}\right\|_{L_{\infty}(t, t+1 ; H)}+\left\|v_{m}\right\|_{L_{2}(t, t+1 ; V)}+\left\|v_{m}^{\prime}\right\|_{L_{4 / 3}\left(t, t+1 ; V^{*}\right)} \leqslant 2 R_{0} .
$$

Покажем, что $v$ принадлежит классу $C\left(\mathbb{R}_{+} ; V^{*}\right) \cap L_{\infty}\left(\mathbb{R}_{+} ; H\right)$. По построению имеем $v \in$ $C\left(\mathbb{R}_{+} ; V^{*}\right)$. Далее, из неравенства (8.8) следует, что для любого отрезка $[t, t+1]$ норма 
vrai $\max _{s \in(t, t+1)}\left\|v_{m}(s)\right\|_{H}=\left\|v_{m}\right\|_{L_{\infty}(t, t+1 ; H)}$ ограничена числом $2 R_{0}$, не зависящим от $m$ и $t$. Следовательно, норма vrai $\max _{s>0}\left\|v_{m}(s)\right\|_{H}=\left\|v_{m}\right\|_{L_{\infty}\left(\mathbb{R}_{+} ; H\right)}$ ограничена тем же числом, и последовательность $\left\{v_{m}\right\}$ сходится к своей предельной функции $v *$-слабо в $L_{\infty}\left(\mathbb{R}_{+} ; H\right)$. Это значит, что предельная функция принадлежит пространству $L_{\infty}\left(\mathbb{R}_{+} ; H\right)$.

Рассмотрим произвольный отрезок $[t, t+1], t \geqslant 0$. Так как $v_{m} \rightarrow v$ в $C\left(\mathbb{R}_{+} ; V^{*}\right)$, то $v_{m} \rightarrow v$ в $C\left(t, t+1 ; V^{*}\right)$. Нормы, стоящие в левой части неравенства (8.8), ограничены, поэтому имеем $v_{m} \rightarrow v *$-слабо в $L_{\infty}(t, t+1 ; H)$ и слабо в $L_{2}(t, t+1 ; V)$. Далее, существует подпоследовательность $v_{m_{k}}^{\prime} \rightarrow u$ слабо в $L_{4 / 3}\left(t, t+1 ; V^{*}\right)$. Однако в смысле распределений $\mathcal{D}^{\prime}\left(t, t+1 ; V^{*}\right)$ имеем $v_{m_{k}}^{\prime} \rightarrow v^{\prime}$. Пользуясь верхней полунепрерывностью нормы в слабой топологии, для предельных функций получаем:

$$
\begin{aligned}
& \|v\|_{L_{\infty}(t, t+1 ; H)}+\|v\|_{L_{2}(t, t+1 ; V)}+\left\|v^{\prime}\right\|_{L_{4 / 3}\left(t, t+1 ; V^{*}\right)} \leqslant \\
& \leqslant \underline{\lim _{k \rightarrow \infty}}\left\|v_{m_{k}}\right\|_{L_{\infty}(t, t+1 ; H)}+\varliminf_{k \rightarrow \infty}\left\|v_{m_{k}}\right\|_{L_{2}(t, t+1 ; V)}+\varliminf_{k \rightarrow \infty}\left\|v_{m_{k}}^{\prime}\right\|_{L_{4 / 3}\left(t, t+1 ; V^{*}\right)} \leqslant \\
& \quad \leqslant \underline{\lim _{k \rightarrow \infty}}\left(\left\|v_{m_{k}}\right\|_{L_{\infty}(t, t+1 ; H)}+\left\|v_{m_{k}}\right\|_{L_{2}(t, t+1 ; V)}+\left\|v_{m_{k}}^{\prime}\right\|_{L_{4 / 3}\left(t, t+1 ; V^{*}\right)}\right) \leqslant 2 R_{0} .
\end{aligned}
$$

Таким образом, $v$ удовлетворяет неравенству (8.7) и, таким образом, принадлежит множеству $P$. Замкнутость и компактность множества $P$ доказаны.

Покажем, что множество $P$ является поглощающим. Пусть $B \subset \mathcal{H}^{+}$множество, ограниченное в $L_{\infty}\left(\mathbb{R}_{+} ; E\right)$. Тогда для $v \in B$ имеем

$$
\|v\|_{L_{\infty}\left(\mathbb{R}_{+} ; E\right)} \leqslant R
$$

Пусть число $h_{0}$ таково, что $2 R^{2} e^{-2 h_{0}} \leqslant 1$. Тогда для $v \in B$ при $h \geqslant h_{0}$ в соответствии с неравенством (8.6) имеем

$$
\begin{aligned}
\|T(h) v\|_{L_{\infty}(t, t+1 ; H)}+\|T(h) v\|_{L_{2}(t, t+1 ; V)}+\left\|T(h) v^{\prime}\right\|_{L_{4 / 3}\left(t, t+1 ; V^{*}\right)} & \\
=\|v\|_{L_{\infty}(h+t, h+t+1 ; H)}+\|v\|_{L_{2}(h+t, h+t+1 ; V)}+\left\|v^{\prime}\right\|_{L_{4 / 3}\left(h+t, h+t+1 ; V^{*}\right)} & \leqslant \\
& \leqslant R_{0}\left(1+2 R^{2} e^{-2 \gamma(t+h)}\right) \leqslant 2 R_{0} .
\end{aligned}
$$

Таким образом, для $T(h) v$ неравенство (8.7) выполняется, и, следовательно, $T(h) B \subset P$ при $h \geqslant h_{0}$. Это значит, что множество $P$ является поглощающим.

Таким образом, множество $P$ является ограниченным в $L_{\infty}\left(\mathbb{R}_{+} ; H\right)$, относительно компактное в $C\left(\mathbb{R}_{+} ; V^{*}\right)$ поглощающее (следовательно, и притягивающее) множество траекторий $\mathcal{H}^{+}$. Таким образом, согласно теореме 2.1 существует минимальный траекторный аттрактор $\mathcal{U}$ пространства траекторий $\mathcal{H}^{+}$.

Существование глобального аттрактора (теорема 2.3) является следствием существования минимального траекторного аттрактора (см. теорему 2.2 и теорему 8.2 ).

\section{СПИСОК ЛИТЕРАТУРЫ}

1. Вишик М. И., Чепыжов В. В. Траекторные аттракторы уравнений математической физики// Усп. мат. наук. - 2011. - 66, № 4 (400). - С. 3-102.

2. Вишик М. И., Чепыжов В. В. Траекторный и глобальный аттракторы 3D системы Навье-Стокса// Мат. заметки. - 2002. - 71, № 2. - С. 194-213.

3. Гаевский X., Грегер K., Захариас K. Нелинейные оператоные уравнения и операторные дифференциальные уравнения. - М.: Мир, 1978.

4. Звягин В. Г., Авдеев Н. Н. Пример системы, минимальный траекторный аттрактор которой не содержит решений системы// Мат. заметки. - 2018. - 104, № 6. - С. 937-941.

5. Звягин В. Г., Дмитриенко В. Т. О слабых решениях начально-краевой задачи для уравнения движения вязкоупругой жидкости// Докл. РАН. - 2001. - 380 (3). - С. 308-311.

6. Звягин В. Г., Дмитриенко В. Т. Аппроксимационно-топологический подход к исследованию задач гидродинамики. Система Навье-Стокса. - М.: Едиториал УРСС, 2004.

7. Звягин В. Г., Кондратъев С. К. Аттракторы слабых решений регуляризованной системы уравнений движения жидких сред с памятью// Мат. сб. - 2012. - 203, № 11. - С. 83-104. 
8. Звягин В. Г., Кондратъев C. К. Аттракторы уравнений неньютоновской гидродинамики// Усп. мат. наук. - 2014. - 69, № 5 (419). - С. 81-156.

9. Ладыюенская О. А. О динамической системе, порождаемой уравнениями Навье-Стокса// Зап. науч. семин. ЛОМИ. - 1972. - 27. - С. 91-114.

10. Ладъженская О.А. О нахождении минимальных глобальных аттракторов для уравнений НавьеСтокса и других уравнений с частными производными // Усп. мат. наук. - 1987. - 42, № 6. - С. 25-60.

11. Литвинов В. Г. Движение нелинейно-вязкой жидкости. - М.: Наука, 1982.

12. Темам Р. Уравнение Навье-Стокса. Теория и численный анализ. - М.: Мир, 1971.

13. Фикера Г. Теоремы существования в теории упругости. - М.: Мир, 1974.

14. Chepyzhov V. V., Vishik M. I. Trajectory attractors for evolution equations// C. R. Acad. Sci. Paris. Ser. I. - 1995. - 321. - P. 1309-1314.

15. Sell G. Global attractors for the three-dimensional Navier-Stokes equations// J. Dynam. Differ. Equations. - 1996. - 8, № 1. - P. 1-3.

16. Sell G. R., You Y. Dynamics of Evolutionary Equations. — New York: Springer-Verlag, 1998.

17. Simon J. Compact sets in $L_{p}(0, T ; B) / /$ Ann. Mat. Pura Appl. Ser. IV. — 1987. — 146. - P. 65-96.

18. Temam R. Infinite-Dimensional Dynamical Systems in Mechanics and Physics. - New York: SpringerVerlag, 1988.

19. Vorotnikov D. A. Asymptotic behaviour of the non-autonomous 3D Navier-Stokes problem with coercive force// J. Differ. Equations. - 2011. - 251. — P. 2209-2225.

20. Vorotnikov D. A., Zvyagin V. G. Trajectory and global attractors of the boundary value problem for autonomous motion equations of viscoelastic medium// J. Math. Fluid Mech. — 2008. — 10. — P. 19-44.

21. Vorotnikov D. A., Zvyagin V. G. Uniform attractors for non-automous motion equations of viscoelastic medium// J. Math. Anal. Appl. — 2007. - 325. - P. 438-458.

22. Zvyagin V. G., Kondratyev S. K. Approximating topological approach to the existence of attractors in fluid mechanics// J. Fixed Point Theory Appl. - 2013. - 13. - P. 359-395.

23. Zvyagin V. G., Vorotnikov D. A. Topological Approximation Methods for Evolutionary Problems of Nonlinear Hydrodynamics. - Berlin-New York: de Gruyter, 2008.

Звягин Виктор Григорьевич

Воронежский государственный университет

E-mail: zvg_vsu@mail.ru

Казначеев Михаил Викторович

Воронежский государственный университет

E-mail: m.v.kaznacheev@yandex.ru 